Received: 14 February 2019

Accepted: 21 May 2019

Published online: 11 June 2019
SCIENTIFIC REP

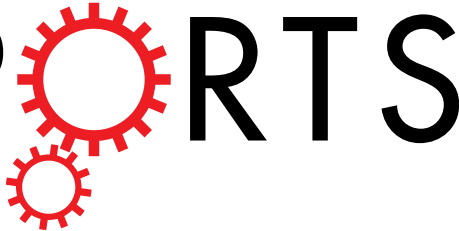

\section{OPEN Chiral magnetic chemical bonds in molecular states of impurities in Weyl semimetals}

\author{
Y. Marques ${ }^{1}$, W. N. Mizobata ${ }^{1}$, R. S. Oliveira ${ }^{1}$, M. de Souza ${ }^{2}$, M. S. Figueira ${ }^{3}$, I. A. Shelykh ${ }^{4,5}$ \& \\ A. C. Seridonio $\mathbb{D}^{1,2}$
}

We demonstrate that chirality of the electron scattering in Weyl semimetals leads to the formation of magnetic chemical bonds for molecular states of a pair of impurities. The effect is associated with the presence of time-reversal symmetry breaking terms in the Hamiltonian which drive a crossover from $s$ - to $p$-wave scattering. The profiles of the corresponding molecular orbitals and their spin polarizations are defined by the relative orientation of the lines connecting two Weyl nodes and two impurities. The magnetic character of the molecular orbitals and their tunability open the way for using doped Weyl semimetals for spintronics and realization of qubits.

Recent years witnessed unprecedented penetration of the ideas of high energy physics into the domain of condensed matter. In particular, lot of attention is now attracted to the condensed matter realizations of three dimensional (3D) massless quasi-relativistic particles known as Dirac or Weyl fermions ${ }^{1}$. The experimental observation of Dirac fermions in such materials as $\mathrm{Na}_{3} \mathrm{Bi}^{2,3}$ and $\mathrm{Cd}_{3} \mathrm{As}_{2}{ }^{4,5}$ made possible the study of the $3 \mathrm{D}$ analogs of graphene physics in a robust topologically protected material possessing both inversion $(\mathcal{I})$ and time $\operatorname{reversal}(\mathcal{T})$ symmetries ${ }^{6}$. In Weyl semimetals, where one of these symmetries is broken, a Dirac node, which is the point where conduction and valence bands touch each other, splits into a pair of Weyl nodes with opposite chiralities. Such nodes are predicted to give rise to a plethora of interesting phenomena, including formation of Fermi arcs, unusual Hall effects, and chiral anomaly, among others ${ }^{6-12}$. The material platform for realization of Weyl fermions is presented by such compounds as tantalum arsenide $(\mathrm{TaAs})^{13-17}$, niobium arsenide $(\mathrm{NbAs})^{18}$, and tantalum phosphide $(\mathrm{TaP})^{19}$.

One of the aspects of Weyl semimetals which recently received particular attention is the peculiar impurity physics $^{20-25}$. For instance, in the case of a single Kondo impurity, Zheng, S.-H. et al. ${ }^{24}$ have observed beating patterns in the local density of states strongly dependent upon the $\mathcal{I}$ and $\mathcal{T}$ symmetries. Additionally, some of us have found an unusual ground state of antibonding-type for a diatomic molecule immersed into a Dirac host, which corresponds to Weyl semimetal with the symmetries above preserved ${ }^{25}$. Thus by working off these regimes, we consider the $\mathcal{T}$ - breaking Weyl semimetal and propose the formation of molecules due to an unprecedented chemical bond mechanism, which we reveal being of chiral-magnetic nature. In the present work, we clarify the role played by chirality of Weyl quasiparticles in the processes of impurity scattering by investigation of the local density of states. The latter can be experimentally addressed by means of the scanning tunneling microscopy (STM). We show that long-range Friedel-like oscillations ${ }^{26}$ contribute to the formation of molecular states in a pair of distant impurities embedded in a 3D relativistic semimetal. We demonstrate that, the scenario of the impurity scattering is radically different in Dirac and Weyl semimetals and show that in the latter case magnetic molecular states can be formed. Their particular type is defined by the relative orientation of the lines connecting two Weyl nodes and two impurities. We report a crossover from $s$ - to $p$-type atomic orbitals for individual impurities and related formation of spin-polarized $\sigma$ - and $\pi$-type molecular orbitals for an impurity pair.

\section{Model}

We set $\hbar=1$ throughout the calculations and represent the total Hamiltonian as the sum of the three terms:

${ }^{1}$ Departamento de Física e Química, Unesp - Univ Estadual Paulista, 15385-000, Ilha Solteira, SP, Brazil. ${ }^{2}$ IGCE, Unesp Univ Estadual Paulista, Departamento de Física, 13506-900, Rio Claro, SP, Brazil. ${ }^{3}$ Instituto de Física, Universidade Federal Fluminense, 24210-340, Niterói, RJ, Brazil. ${ }^{4}$ Science Institute, University of Iceland, Dunhagi-3, IS-107, Reykjavik, Iceland. ${ }^{5}$ ITMO University, St. Petersburg, 197101, Russia. Correspondence and requests for materials should be addressed to A.C.S. (email: antonio.seridonio@unesp.br) 


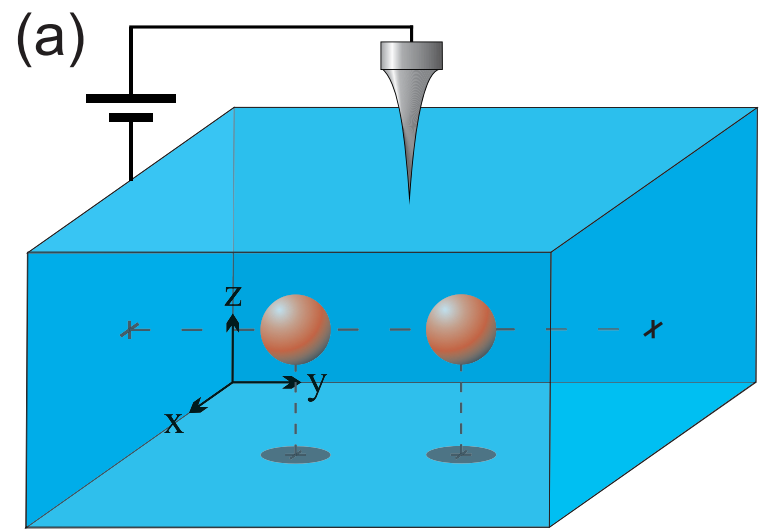

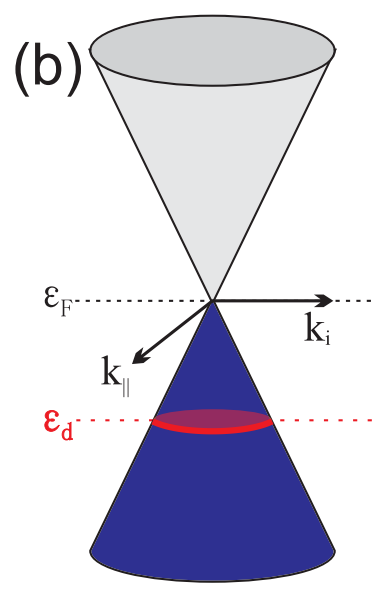

Dirac
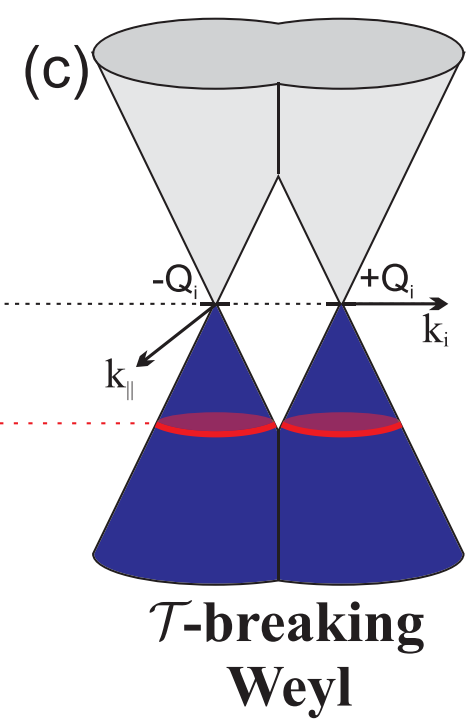

Figure 1. Panel (a) Sketch of the proposed setup. Two impurities are embedded in a 3D semimetal of Dirac or Weyl type. The density of electrons forming molecular orbitals can be probed by an STM-tip. Panels (b,c) show low energy band structure for Dirac and $\mathcal{T}$-breaking Weyl semimetals with two Weyl nodes located at $\pm Q_{i}$, $i=x, y, z$. The blue color of the lower cones indicates the filling of the valence bands, black dotted line is the Fermi energy set at $\varepsilon_{F}=0$ and red dotted line corresponds to the single-particle energy of the impurities.

$$
\mathcal{H}=\mathcal{H}_{0}+\mathcal{H}_{\mathrm{d}}+\mathcal{H}_{\mathcal{V}}
$$

The low-energy Hamiltonian of the host may be represented as

$$
\mathcal{H}_{0}=\sum_{\mathbf{k}} \psi^{\dagger}(\mathbf{k})\left(H_{+} \oplus H_{-}\right) \psi(\mathbf{k})
$$

where $\psi(\mathbf{k})=\left(c_{\mathbf{k}+\uparrow}, c_{\mathbf{k}+\downarrow}, c_{\mathbf{k}-\uparrow}, c_{\mathbf{k}-\downarrow}\right)^{T}$ is four-spinor operator whose components $c_{\mathbf{k} \chi \sigma}^{\dagger}\left(c_{\mathbf{k} \chi \sigma}\right)$ stand for the creation (annihilation) operators of an electron with wave number $k$ and spin $\sigma$,

$$
H_{\chi}(\mathbf{k})=\chi v_{F} \sigma \cdot(\mathbf{k}-\chi Q),
$$

where $\mathbf{k}=\left(k_{x}, k_{y}, k_{z}\right)$ is the three-dimensional wave vector, $\sigma$ stands for the vector of Pauli matrices, the index $\chi= \pm 1$ corresponds to the chirality of the Weyl nodes and $v_{F}$ is the Fermi velocity. For $Q=0, \mathcal{T}$ symmetry is conserved and a pair of Weyl nodes is degenerated, which corresponds to the case of a standard Dirac semimetal. If $\mathcal{T}$ symmetry is broken $(Q \neq 0)$, two Weyl nodes are displaced with respect to each other towards two different points in the Brillouin zone located at $\pm Q$, but maintain energetic degeneracy as it is depicted in the Fig. 1 .

The impurities are modeled by the Hamiltonian

$$
\mathcal{H}_{\mathrm{d}}=\sum_{j \sigma} \varepsilon_{j \sigma} d_{j \sigma}^{\dagger} d_{j \sigma}+\sum_{j} U_{j} n_{j \uparrow} n_{j \downarrow},
$$



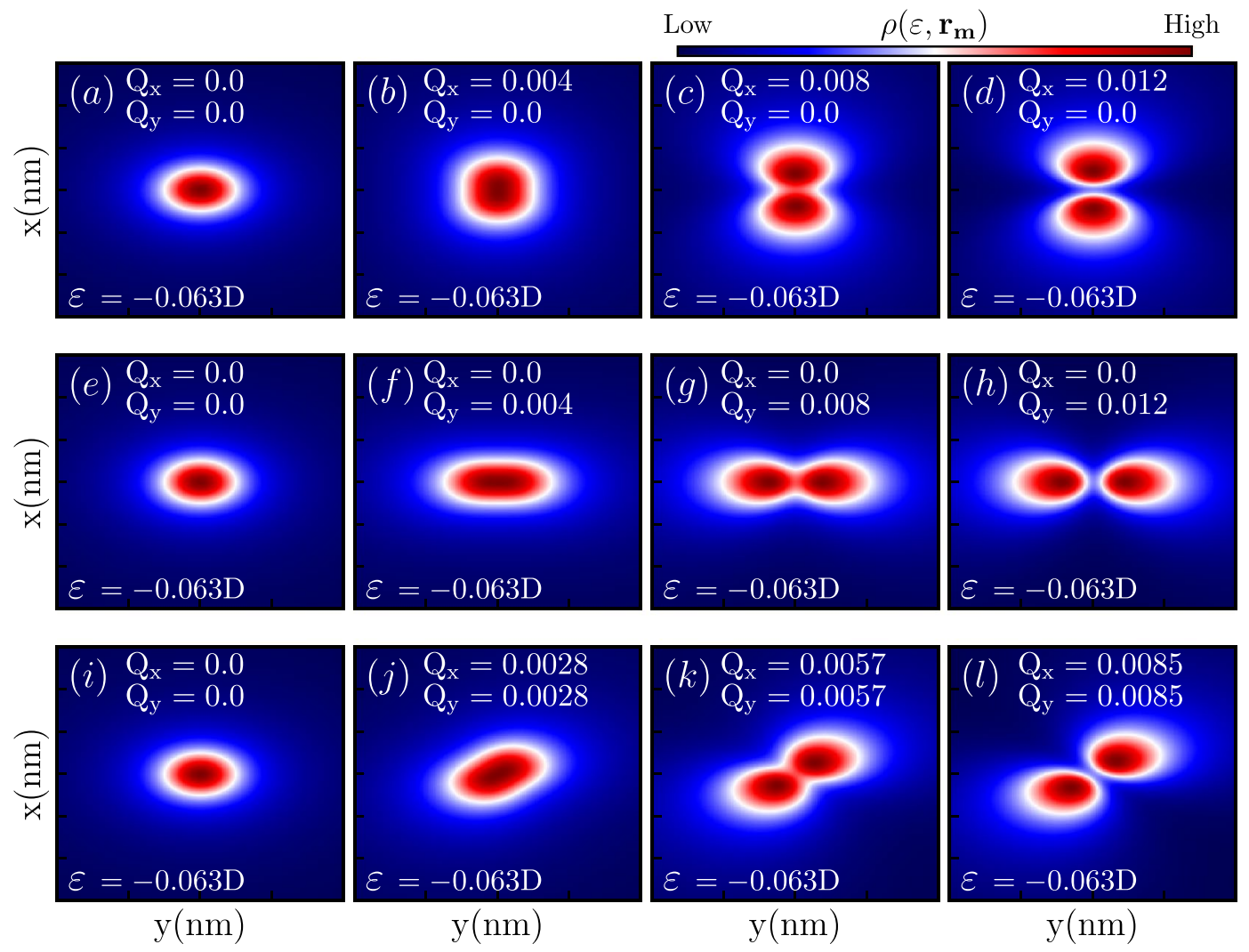

Figure 2. 2D LDOS maps for the case of a single impurity taken at fixed energy $\varepsilon$. Crossover from $s$ - to $p$-type orbitals associated with moving from $\operatorname{Dirac}(Q=0)$ to Weyl $(Q \neq 0)$ regime is clearly seen.

with $\varepsilon_{j \sigma}$ being single-particle energy and $U_{j}$ the on-site Coulomb repulsion, whereas $n_{j \sigma}=d_{j \sigma}^{\dagger} d_{j \sigma}$ corresponds to the number of electrons with spin projection $\sigma$ at the site $j$ with $d_{j \sigma}^{\dagger}$ and $d_{j \sigma}$ being respectively creation and annihilation operators.

The hybridization between the host and the impurities is described by the term:

$$
\mathcal{H}_{\mathcal{V}}=\sum_{j \mathbf{k}} \hat{d}_{j}^{\dagger} \hat{V}_{j \mathbf{k}} \psi(\mathbf{k})+\text { H. c. }
$$

wherein $\hat{d}_{j}^{\dagger}=\left(\begin{array}{ll}d_{j \uparrow}^{\dagger}, & d_{j \downarrow}^{\dagger}\end{array}\right)$ and

$$
\hat{V}_{j \mathbf{k}}=\left(\begin{array}{llll}
V_{j \mathbf{k}} & 0 & V_{j \mathbf{k}} & 0 \\
0 & V_{j \mathbf{k}} & 0 & V_{j \mathbf{k}}
\end{array}\right),
$$

where $V_{j \mathbf{k}}=\frac{v_{0}}{\sqrt{N}} e^{i \mathbf{k} \cdot \mathbf{R}_{j}}$, with $\nu_{0}$ being the hybridization amplitude between electrons of the host and localized states of the impurities positioned at $\mathbf{R}_{j}(j=1,2), N$ is the normalization factor yielding the total number of the conduction states. As performed by some of $\mathrm{us}^{27}$, we assume for a sake of simplicity, that the hopping term $V_{j \mathbf{k}}$ neglects the exponential decay of the Bloch states right above the Weyl semimetal surface that overlap with those from the STM-tip apex. By employing such an assumption, STM-tip measurements of the local density of states would be just attenuated with respect to the values extracted from our simulations, in such a way that none generality is lost as ensured by Plihal, M. et al. ${ }^{28}$, which have analyzed this density for the single-impurity problem.

\section{Local Density of States (LDOS)}

The electronic properties of the considered system are determined by the LDOS of the host which can be experimentally accessed by means of an STM-tip. It can be calculated using standard equation-of-motion (EOM) procedure $^{29,30}$ as:

$$
\rho\left(\varepsilon, \mathbf{r}_{m}\right)=-\frac{1}{\pi} \sum_{\sigma} \operatorname{Im}\left\{\widetilde{\mathcal{G}}_{\sigma}\left(\varepsilon, \mathbf{r}_{m}\right)\right\}=\rho_{0}(\varepsilon)+\sum_{j j^{\prime}} \delta \rho_{j j^{\prime}}\left(\varepsilon, \mathbf{r}_{m}\right),
$$

where $\widetilde{\mathcal{G}}_{\sigma}\left(\varepsilon, \mathbf{r}_{m}\right)$ is the time-Fourier transform of the retarded Green's function in the time domain, defined as: 


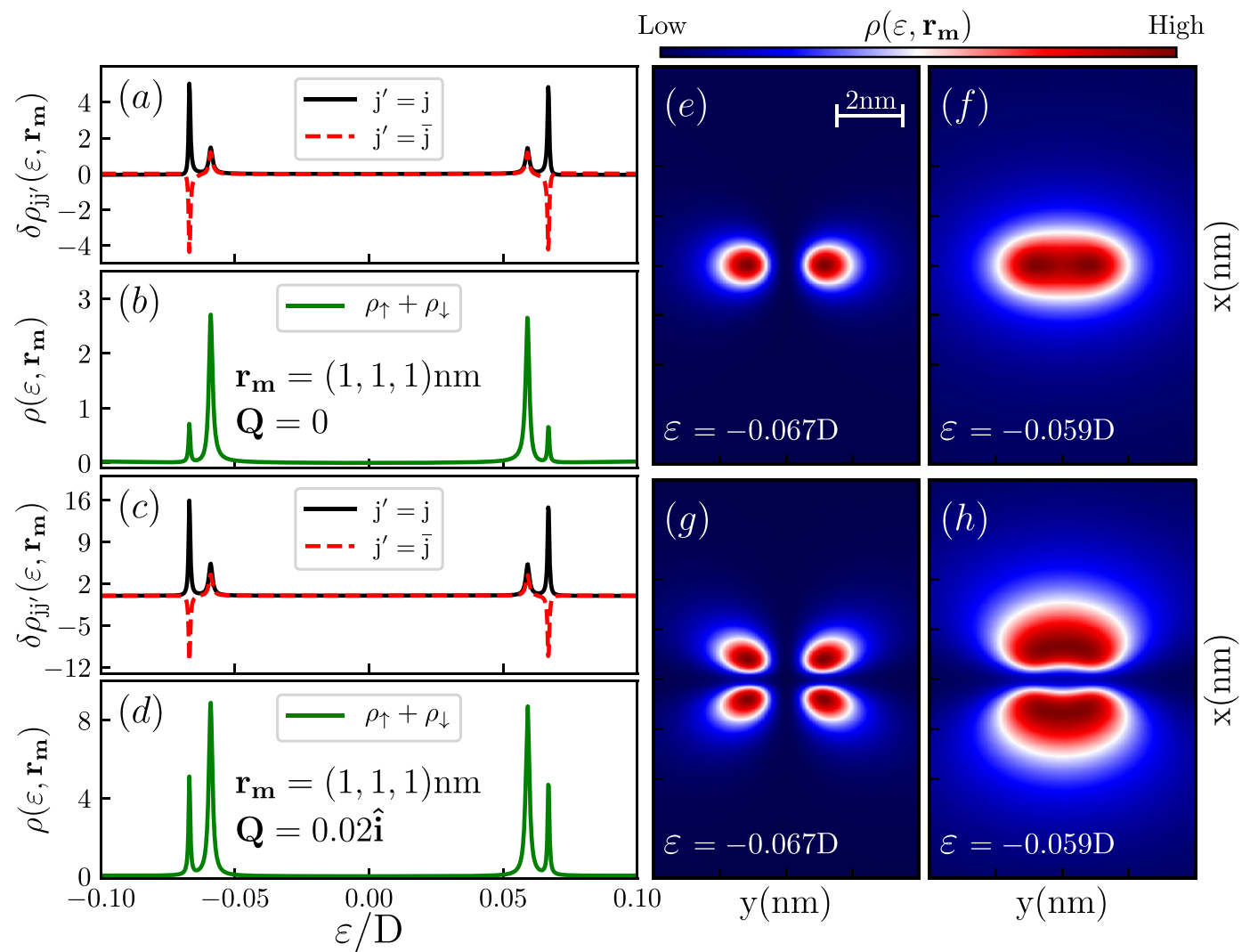

(i)

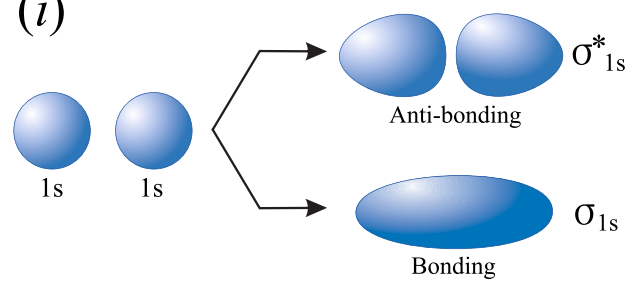

$(j)$

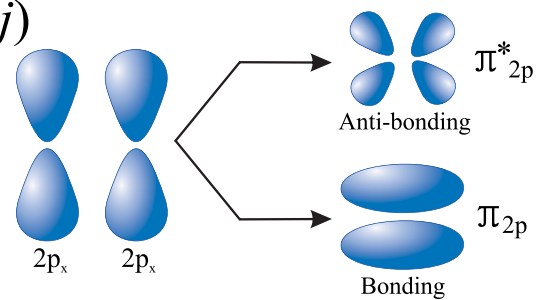

Figure 3. LDOS for a pair of impurities. The panels (a,b,e,f) correspond to the case of Dirac semimetal $(Q=0)$, the panels $(\mathrm{c}, \mathrm{d}, \mathrm{g}, \mathrm{h})$ to the case of $\mathcal{T}$-breaking Weyl semimetal with $Q \cdot r_{12}=0$. Panels (a and c) display the diagonal $\left(\delta \rho_{i j}\right)$ and off-diagonal $\left(\delta \rho_{i j}\right)$ contributions to the LDOS. Note that $\delta \rho_{i j}$ reveals both dips and peaks corresponding to anti-resonances and resonances respectively, while $\delta \rho_{j j}$ reveals peaks only. The total LDOS is presented in the panels ( $\mathrm{b}$ and $\mathrm{d}$ ). We set $\mathbf{r}_{m}=(1,1,1) \mathrm{nm}$, the energy is counted from the Fermi level set at $\varepsilon_{F}=0$, for the Weyl host $Q_{x}=0.02$. The total LDOS on the $\mathbf{r}_{m}=(x, y, 1) \mathrm{nm}$ surface for the energies of bonding and antibonding states $(\varepsilon=-0.067 \mathrm{D}$ and $\varepsilon=-0.059 \mathrm{D})$ is shown in panels (e,f,g,h). Panels (i and $\mathrm{j}$ ) illustrate how molecular orbitals presented in the panels $(\mathrm{e}-\mathrm{h})$ are formed from atomic orbitals presented in the Fig. 2.

$$
\mathcal{G}_{\sigma}\left(t, \mathbf{r}_{m}\right)=-i \theta(t)\left\langle\left\{\psi_{\sigma}\left(t, \mathbf{r}_{m}\right), \psi_{\sigma}^{\dagger}\left(0, \mathbf{r}_{m}\right)\right\}\right\rangle_{\mathcal{H}},
$$

where $\theta(t)$ denotes the Heaviside function, $\psi_{\sigma}\left(t, \mathbf{r}_{m}\right)$ is the field operator of the host electrons written in terms of the continuous variable $\mathbf{r}_{m}$, the brackets $\langle\cdots\rangle_{\mathcal{H}}$ denote the ensemble average with respect to the full Hamiltonian, $\{\cdots\}$ determines an anti-commutator between operators in the Heisenberg picture, $\rho_{0}(\varepsilon)=\frac{6 \varepsilon^{2}}{D^{3}}$ is the pristine host DOS with $D$ being the energy cutoff corresponding to the half-bandwidth, and

$$
\delta \rho_{j j^{\prime}}(\varepsilon)=-\frac{1}{\pi v_{0}^{2}} \sum_{\chi \chi^{\prime}} \sum_{\sigma} \operatorname{Im}\left[\Sigma_{\sigma}^{\chi}\left(\mathbf{r}_{m j}\right) \widetilde{G}_{j \sigma \mid j^{\prime} \sigma}(\varepsilon) \Sigma_{\sigma}^{\chi^{\prime}}\left(\mathbf{r}_{j^{\prime} m}\right)\right]
$$

encodes the Friedel-like oscillations describing the scattering of the conduction electrons by the impurities, where the terms $j^{\prime}=j$ and $j^{\prime} \neq j$ give rise to intra and inter-impurity scattering processes, respectively, which are ruled by the spatial dependent self-energy 

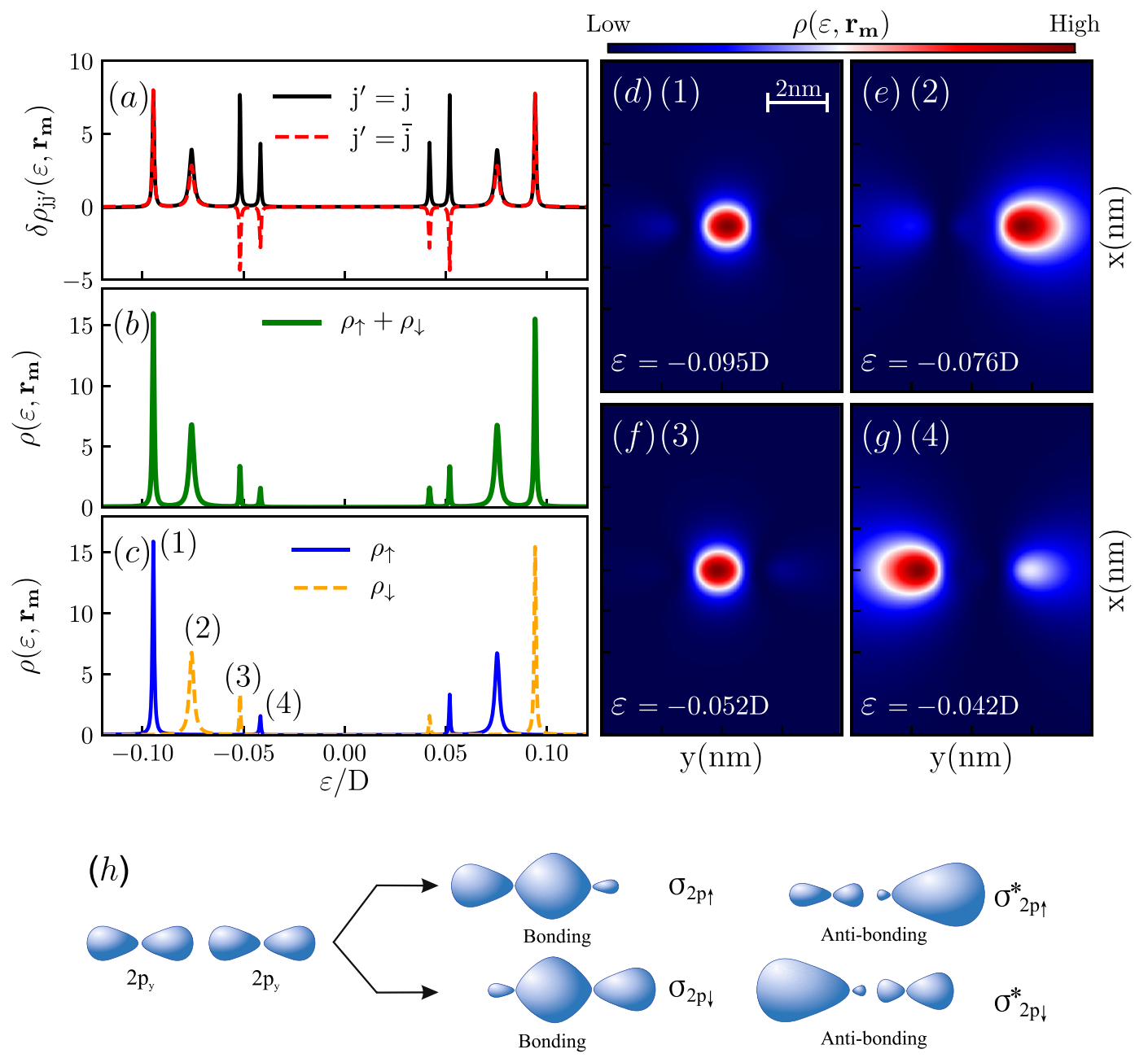

Figure 4. LDOS for the $\mathcal{T}$-breaking Weyl semimetal for the case when vectors $Q$ and $r_{12}$ are parallel (we took $Q=0.02 \hat{j})$. Panel (a) displays diagonal $\left(\delta \rho_{j j}\right)$ and off-diagonal $\left(\delta \rho_{\overline{j j}}\right)$ contributions to the LDOS. The total LDOS is presented in the panel (b). Panel (c) shows spin-resolved density of states. The map of the total LDOS on the $\mathbf{r}_{m}=(x, y, 1) \mathrm{nm}$ surface for the energies corresponding to the four spin-resolved molecular states is presented in the panels (d-g). Panel (h) illustrates how molecular orbitals presented in the panels $(\mathrm{d}-\mathrm{g})$ are formed from atomic orbitals presented in the Fig. 2.

$$
\Sigma_{\sigma}^{\chi}\left(\mathbf{r}_{m j}\right)=-\frac{3 v_{0}^{2} \pi v_{F}}{2 D^{3}\left|\mathbf{r}_{m j}\right|} e^{-i\left|\mathbf{r}_{m j}\right| \frac{\varepsilon}{V_{F}}} e^{-i \chi \mathbf{Q} \cdot \mathbf{r}_{m j}}\left(\varepsilon \pm \chi \sigma \varepsilon \pm i \frac{\chi \sigma v_{F}}{\left|\mathbf{r}_{m j}\right|}\right),
$$

$\mathbf{r}_{m j}=\mathbf{r}_{m}-\mathbf{R}_{j}$ and \pm signs correspond to the vector direction (positive for $\mathbf{r}_{m j}$, negative for $\mathbf{r}_{j m}$ ). The Eq. (10) is spatially anisotropic and the LDOS as a result, due to mutual orientation of $Q$ and $\mathbf{r}_{m j}$ inside the plane wave $e^{-i \chi Q \cdot \mathbf{r}_{m j}}$. Consequently, it leads to the main finding of this work, which is ruled by the mixing of chirality $(\chi)$ and spin $(\sigma)$ quantum numbers, as one can perceive by the presence of the crossed terms $\pm \chi \sigma \varepsilon \pm i \frac{\chi \sigma v_{F}}{\left|\mathbf{r}_{m j}\right|}$. Such a set of terms, thus gives rise to the possibility of spin-polarized molecular states, which are chiral-dependent as we will see below. To show the emergence of such, we should regard the system anisotropy by means of the condition $e^{-i \chi Q \cdot \mathbf{r}_{j j^{\prime}}} \neq 1$, with the relative distance between the impurities given by $\mathbf{r}_{j j^{\prime}}=\mathbf{R}_{j}-\mathbf{R}_{j^{\prime}}$. As aftermath, the spin degree of freedom in the quasiparticle energy correction of the impurity $j$ due to $j^{\prime}$ (and vice-versa), namely $\Sigma_{\sigma}\left(\mathbf{r}_{j j^{\prime}}\right)=\sum_{\chi} \Sigma_{\sigma}^{\chi}\left(\mathbf{r}_{j j^{\prime}}\right)$, becomes lifted. In this way, $\Sigma_{\uparrow}\left(\mathbf{r}_{j j^{\prime}}\right) \neq \Sigma_{\downarrow}\left(\mathbf{r}_{j j^{\prime}}\right)$ and spin-polarized molecular states are allowed. In the absence of anisotropy $\left(e^{-i \chi \mathbf{Q} \cdot \mathbf{r}_{j j^{\prime}}}=1\right)$, the degeneracy $\Sigma_{\uparrow}\left(\mathbf{r}_{j j^{\prime}}\right)=\Sigma_{\downarrow}\left(\mathbf{r}_{j j^{\prime}}\right)$ holds and paramagnetic molecular states prevail. We should stress that, such an effect is entirely distinct from the corresponding caused by an external Zeeman filed. In the latter, it breaks naturally the spin-degeneracy whatever the orientation $\mathbf{Q} \cdot \mathbf{r}_{j i}$ ' Thus, the addressed chiral magnetic effect reveals that an applied magnetic field is not capable of reproducing this peculiar magnetism and prevents the feasibility of the proposed chiral magnetic chemical bond as well. We guide the reader to the supplementary material, where a concise derivation of Eq. (10) can be readily followed.

$\widetilde{\mathcal{G}}_{j \sigma \mid j^{\prime} \sigma}(\varepsilon)$ is the time-Fourier transform of the Green's function of the impurities 

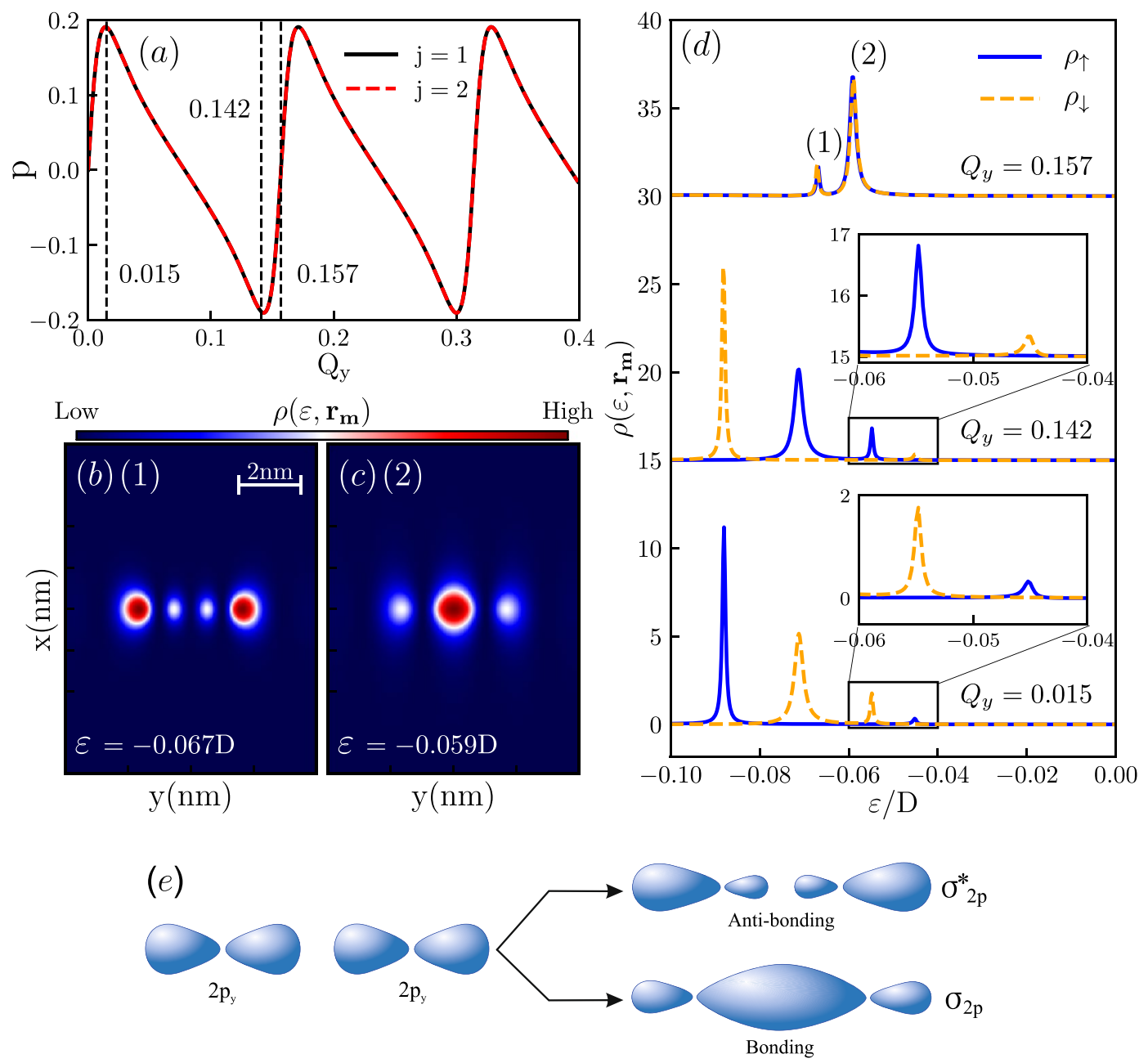

Figure 5. Panel (a) Total magnetization of the impurities as function of the parameter $Q_{y}$ describing the shift of the Weyl nodes in the reciprocal space in the direction parallel to the line connecting impurities. Panels $(b, c)$ The maps of the total LDOS on the $\mathbf{r}_{m}=(x, y, 1) \mathrm{nm}$ surface for the energies corresponding to bonding and antibonding states in the valence band for the spin degenerate case corresponding to $Q_{y}=0.157$. Panel (d) Spin resolved LDOS at the point $\mathbf{r}_{m}=(1,1,1) \mathrm{nm}$ for spin degenerate case $\left(Q_{y}=0.157\right)$ and maximal spin up $\left(Q_{y}=0.015\right)$ and spin down $\left(Q_{y}=0.142\right)$ polarizations. The LDOS for $Q_{y}=0.157$ is rescaled by the factor of 5 . The insets highlight the marked sectors. Panel (e) illustrates how molecular orbitals presented in the panels (b and c) are formed from atomic orbitals presented in the Fig. 2.

$$
\mathcal{G}_{j \sigma \mid j^{\prime} \sigma}=-i \theta(t)\left\langle\left\{d_{j \sigma}(t), d_{j^{\prime} \sigma}^{\dagger}(0)\right\}\right\rangle_{\mathcal{H}} .
$$

Application of the EOM method to $\widetilde{\mathcal{G}}_{j \sigma \mid j^{\prime} \sigma}(\varepsilon)$ together with Hubbard-I decoupling scheme ${ }^{31}$, yields

$$
\widetilde{\mathcal{G}}_{j \sigma \mid j \sigma}(\varepsilon)=\frac{\lambda_{j}^{\bar{\sigma}}}{g_{j \sigma \mid j \sigma}^{-1}(\varepsilon)-\lambda_{j}^{\bar{\sigma}} \Sigma_{\sigma}\left(\mathbf{r}_{j j^{\prime}}\right) g_{j^{\prime} \sigma \mid j^{\prime} \sigma}(\varepsilon) \lambda_{j^{\prime}}^{\bar{\sigma}} \Sigma_{\sigma}\left(\mathbf{r}_{j^{\prime} j}\right)},
$$

where $\bar{\sigma}=-\sigma, j \neq j^{\prime}, \lambda_{j}^{\bar{\sigma}}=1+\frac{U_{j}}{g_{j \sigma \mid j \sigma}^{-1}(\varepsilon)-U_{j}}\left\langle n_{j \bar{\sigma}}\right\rangle$ is the spectral weight, $g_{j \sigma \mid j \sigma}(\varepsilon)=\frac{1}{\varepsilon-\varepsilon_{j \sigma}-\Sigma_{0}}$ as the single impurity noninteracting Green's function,

$$
\left\langle n_{j \bar{\sigma}}\right\rangle=-\frac{1}{\pi} \int_{-\infty}^{+\infty} n_{F}(\varepsilon) \operatorname{Im}\left(\widetilde{\mathcal{G}}_{j \bar{\sigma} \mid j \bar{\sigma}}(\varepsilon)\right) d \varepsilon
$$

is the occupation number of an impurity with $n_{F}(\varepsilon)$ being the Fermi-Dirac distribution,

$$
\Sigma_{0}=\frac{3 v_{0}^{2}}{D^{2}}\left(\frac{\varepsilon^{2}}{D} \ln \left|\frac{D+\varepsilon}{D-\varepsilon}\right|-2 \varepsilon-i \frac{\varepsilon^{2}}{D}\right)
$$

is the local self-energy and 


$$
\widetilde{\mathcal{G}}_{j \sigma \mid j \sigma}(\varepsilon)=g_{j \sigma \mid j \sigma}(\varepsilon) \lambda_{j}^{\bar{\sigma}} \Sigma_{\sigma}\left(\mathbf{r}_{j j^{\prime}} \tilde{\mathcal{G}}_{j^{\prime} \sigma \mid j^{\prime} \sigma}(\varepsilon)\right.
$$

\section{Results and Discussion}

In order to understand the formation of the molecular states of a pair of impurities inside a Weyl semimetal, we should start from analyzing the case of a single impurity. As model parameters, we adopt the energy of an impurity $\varepsilon_{j \sigma}=-0.07 D$, hybridization amplitude $\nu_{0}=-0.14 D$, on-site Coulomb repulsion $U_{j}=0.14 D, \hbar v_{F} \approx 3 \mathrm{eVA}$, $D \approx 0.2 \mathrm{eV}$ and temperature $T=0 \mathrm{~K}$. Concerning this latter, we clarify that none generality is lost, once finite $T$ just introduces thermal broadening into the Fermi-Dirac distribution $n_{F}(\varepsilon)$ as well as in the LDOS via Eq. (13) for the impurity occupation number $\left\langle n_{j \sigma}\right\rangle$ and, according to some of us have ${ }^{27}$, phonon modes rise as effective by increasing $T$ and restore the molecular ground state to bonding-type. Thus in the current work, the molecules show antibonding ground state ${ }^{25}$, due to the $T=0 \mathrm{~K}$ condition.

As one can see in the Fig. 2, the 2D map of the LDOS which can be probed by an STM-tip over the system surface presents a crossover from $s$ - to $p$-type atomic orbitals as $Q$ is increased and one moves from $\operatorname{Dirac}(Q=0)$ towards the Weyl regime $(Q \neq 0)$. This happens due to the presence of the terms depending on $v_{F} \chi Q$ in the original Hamiltonian. Note, that the $p$-orbital is elongated along the direction of $Q$.

Now we can analyze the molecular state corresponding to a pair of impurities inside a Weyl semimetal with broken $\mathcal{T}$-symmetry. We will consider two cases of the mutual orientation of the vectors $Q$ and $r_{12}=R_{1}-R_{2}$ connecting the two impurities: (i) perpendicular orientation, $Q \cdot r_{12}=0$ and (ii) parallel orientation $Q \cdot r_{12}=|Q|\left|r_{12}\right|$. As we will demonstrate, the former case corresponds to the formation of spin degenerate molecular orbitals, while the latter case gives rise to the chiral magnetic chemical bonds. In all plots of $\rho\left(\varepsilon, \mathbf{r}_{m}\right)$ and $\delta \rho_{j j^{\prime}}\left(\varepsilon, \mathbf{r}_{m}\right)$ versus $\varepsilon / D$ we present, the STM-tip is pinned at the site $\mathbf{r}_{m}=(1,1,1) \mathrm{nm}$ right above the Weyl semimetal surface and the impurities are buried into the bulk at $\mathbf{R}_{1,2}=(0, \mp 1,0) \mathrm{nm}$.

Let us start from the case $Q \cdot r_{12}=0$. In the case of an individual impurity, a single energy resonance appears within the valence band in $\rho\left(\varepsilon, \mathbf{r}_{m}\right)$. Naturally, in the two-impurity system a pair of peaks corresponding to bonding and antibonding states appears, as it is shown in the Fig. $3 \mathrm{~b}$ and $\mathrm{d}$ for the cases of Dirac $(Q=0)$ and $\mathcal{T}$ -breaking Weyl $(Q \neq 0)$ hosts. Note, that the coupling between the impurities is fully mediated by Friedel-like oscillations of the electronic density of the host mathematically described by the self-energy $\lambda_{j}^{\bar{\sigma}} \Sigma_{\sigma}\left(\mathbf{r}_{j j}\right) g_{\bar{j} \sigma \mid \bar{j} \sigma}(\varepsilon) \lambda_{\bar{j}}^{\bar{\sigma}} \Sigma_{\sigma}\left(\mathbf{r}_{\bar{j} j}\right)$ entering into the denominator of $\widetilde{\mathcal{G}}_{j \sigma \mid j \sigma}(\varepsilon)$ given by the Eq. (12).

The 2D map of the molecular orbitals on the host surface is presented in the panels (e-h) of the Fig. 3. Panels (e and f) correspond to the case of a Dirac host which was previously considered by some of us in the ref. ${ }^{25}$. One clearly sees the emergence of bonding and antibonding molecular orbitals with $\sigma$-type symmetry resulting from the interference between two $s$-wave atomic orbitals of individual impurities, as it is illustrated in the panel (i). Panels ( $\mathrm{g}$ and $\mathrm{h}$ ) correspond to the case of a Weyl semimetal with $Q \cdot \mathbf{r}_{\overline{i j}}=0$, for which individual impurities reveal $p$-type atomic orbitals stretched in the direction perpendicular to the line connecting the impurities. Note, that for the considered case bonding and antibonding molecular orbitals have clear $\pi$-type symmetry. These orbitals remain spin-degenerate, as it follows from the Eq. (10), which leads to $\Sigma_{\sigma}\left(\mathbf{r}_{j j^{\prime}}\right)=\sum_{\chi} \Sigma_{\sigma}^{\chi}\left(\mathbf{r}_{j j^{\prime}}\right)$ independent of the spin degree of freedom, i.e., $\Sigma_{\uparrow}\left(r_{i j^{\prime}}\right)=\Sigma_{\downarrow}\left(r_{j j^{\prime}}\right)$.

The case of the parallel orientation of the vectors $Q$ and $\mathbf{r}_{12}$ is illustrated by the Fig. 4 . Note that in this case, according to the Eq. (10) the presence of the terms $e^{i \chi Q \cdot \mathbf{r}_{j j}}$ with $\chi= \pm 1$ in the expression for the self-energy $\Sigma_{\sigma}\left(\mathbf{r}_{j \bar{j}}\right)=\sum_{\chi} \Sigma_{\sigma}^{\chi}\left(\mathbf{r}_{\bar{j}}\right)$, leads to the lifting of spin degeneracy and gives rise to the formation of chiral magnetic chemical bonds. Interestingly enough, this spin-dependency can not be considered as being fully equivalent to one induced by effective external magnetic field, once the sequence of the peaks in the LDOS presented in the Fig. 4(b) does not correspond to the alternation of spin-up and spin-down states as usual, but consists of the two inner spin-down states flanked by the two outer spin-up states as can be clearly seen from the Fig. 4(c). The profiles of the spin-resolved orbitals corresponding to the bonding and antibonding states are shown in the panels (d-g) of the Fig. 4. These orbitals exhibit $\sigma$-type symmetry and are formed due to the interference between two frontal $p$-wave orbitals as sketched in the Fig. $4(\mathrm{~h})$.

To shed more light on the splitting between spin-polarized components in the LDOS, we investigate the impurity magnetization characterized by the polarization degree $p=\left(\left\langle n_{j \uparrow}\right\rangle-\left\langle n_{j \downarrow}\right\rangle\right) /\left(\left\langle n_{j \uparrow}\right\rangle+\left\langle n_{j \downarrow}\right\rangle\right)$, where the occupation numbers are defined by the Eq. (13). The dependence of the magnetization on the separation between the Weyl nodes in the direction parallel to the line connecting the two impurities $Q_{y}$ is shown in the Fig. 5(a). One clearly sees pronounced periodic behavior, stemming from the oscillations of the factor $e^{i \chi Q \cdot \mathbf{r}_{j j}}$ in the expression for spin-resolved self-energy in the Eq. (10). The LDOS corresponding to the spin-degenerate case, maximal positive and negative magnetizations, is shown in the Fig. 5(d). Note that for the spin-degenerate situation corresponding to $Q_{y}=0.157$, the shape of the molecular orbitals presented in the Fig. 5(b,c) can be represented as linear combination of the orbitals presented in the Fig. 4(d-g).

\section{Conclusions}

We analyzed the structure of the molecular orbitals corresponding to the pair of impurities placed within a Weyl semimetal focusing on the role played by the $\mathcal{T}$-symmetry breaking. For this purpose the corresponding LDOS was evaluated. It was demonstrated that the terms in the self-energy stemming from the chiral dependent minimal coupling drive a crossover from spin degenerate $\sigma$-type molecular orbitals characteristic for the case of a Dirac host to spin degenerate $\pi$-type orbitals or spin-polarized $\sigma$-type orbitals for the case of a Weyl host. The type of the chemical bonding in this latter case can be controlled by variation of the mutual position of the impurities with respect to the 
vector $Q$ describing the shift of the Weyl nodes. The magnetic character of the molecular orbitals and their tunability open the way for using doped Weyl semimetals for spintronics and realization of qubits.

\section{Methods}

The findings of this research depicted in the figures of section Results and Discussion, which concern the Eq. (7) for the system LDOS, were obtained by performing self-consistent evaluations of the occupation numbers defined by the Eq. (13) for the impurities. Such calculations and the aforementioned figures were performed by using numerical packages in Python version 3.7.

\section{Data Availability}

The authors declare that the data supporting the findings of this study are available within the paper (and its supplementary information files).

\section{References}

1. Weyl, H. Elektron und gravitation. i. Zeitschrift für Physik 56, 330-352, https://doi.org/10.1007/BF01339504 (1929).

2. Wang, Z. et al. Dirac semimetal and topological phase transitions in $\mathrm{A}_{3} \mathrm{Bi}(\mathrm{A}=\mathrm{Na}, \mathrm{K}, \mathrm{Rb})$. Phys. Rev. B 85, 195320, https://doi. org/10.1103/PhysRevB.85.195320 (2012).

3. Liu, Z. K. et al. Discovery of a three-dimensional topological Dirac semimetal, $\mathrm{Na}_{3} \mathrm{Bi}$. Science 343, 864-867, https://doi.org/10.1126/ science.1245085 (2014).

4. Wang, Z., Weng, H., Wu, Q., Dai, X. \& Fang, Z. Three-dimensional Dirac semimetal and quantum transport in Cd ${ }_{3}$ s $_{2}$. Phys. Rev. B 88, 125427, https://doi.org/10.1103/PhysRevB.88.125427 (2013).

5. Liu, Z. K. et al. A stable three-dimensional topological Dirac semimetal $\mathrm{Cd}_{3} \mathrm{As}_{2}$. Nature Materials 13, 677 EP- (2014).

6. Armitage, N. P., Mele, E. J. \& Vishwanath, A. Weyl and Dirac semimetals in three-dimensional solids. Rev. Mod. Phys. 90, 015001, https://doi.org/10.1103/RevModPhys.90.015001 (2018).

7. Wan, X., Turner, A. M., Vishwanath, A. \& Savrasov, S. Y. Topological semimetal and Fermi-arc surface states in the electronic structure of pyrochlore iridates. Phys. Rev. B 83, 205101, https://doi.org/10.1103/PhysRevB.83.205101 (2011).

8. Yang, K.-Y., Lu, Y.-M. \& Ran, Y. Quantum hall effects in a Weyl semimetal: Possible application in pyrochlore iridates. Phys. Rev. B 84, 075129, https://doi.org/10.1103/PhysRevB.84.075129(2011).

9. Hosur, P., Parameswaran, S. A. \& Vishwanath, A. Charge transport in Weyl semimetals. Phys. Rev. Lett. 108, 046602, https://doi. org/10.1103/PhysRevLett.108.046602 (2012).

10. Kim, P., Ryoo, J. H. \& Park, C.-H. Breakdown of the chiral anomaly in Weyl semimetals in a strong magnetic field. Phys. Rev. Lett. 119, 266401, https://doi.org/10.1103/PhysRevLett.119.266401 (2017)

11. Nielsen, H. \& Ninomiya, M. The adler-bell-jackiw anomaly and Weyl fermions in a crystal. Physics Letters B 130, 389-396, https:// doi.org/10.1016/0370-2693(83)91529-0 (1983).

12. Xu, G., Weng, H., Wang, Z., Dai, X. \& Fang, Z. Chern semimetal and the quantized anomalous hall effect in $\mathrm{HgCr}_{2} \mathrm{Se}_{4}$. Phys. Rev. Lett. 107, 186806, https://doi.org/10.1103/PhysRevLett.107.186806 (2011).

13. Huang, S.-M. et al. A Weyl fermion semimetal with surface Fermi arcs in the transition metal monopnictide TaAs class. Nature Communications 6, $7373 \mathrm{EP}-(2015)$.

14. Weng, H., Fang, C., Fang, Z., Bernevig, B. A. \& Dai, X. Weyl semimetal phase in noncentrosymmetric transition-metal monophosphides. Phys. Rev. X 5, 011029, https://doi.org/10.1103/PhysRevX.5.011029 (2015).

15. Xu, S.-Y. et al. Discovery of a Weyl fermion semimetal and topological Fermi arcs. Science 349, 613-617, https://doi.org/10.1126/ science.aaa9297 (2015).

16. Lv, B. Q. et al. Experimental discovery of Weyl semimetal TaAs. Phys. Rev. X 5, 031013, https://doi.org/10.1103/PhysRevX.5.031013 (2015).

17. Lv, B. Q. et al. Observation of Weyl nodes in TaAs. Nature Physics 11, 724 EP- (2015).

18. Xu, S.-Y. et al. Observation of Fermi arc surface states in a topological metal. Science 347, 294-298, https://doi.org/10.1126/ science.1256742 (2015).

19. Xu, N. et al. Observation of Weyl nodes and Fermi arcs in tantalum phosphide. Nature Communications 7, 11006 EP- (2016).

20. Sun, J.-H., Xu, D.-H., Zhang, F.-C. \& Zhou, Y. Magnetic impurity in a Weyl semimetal. Phys. Rev. B 92, 195124, https://doi. org/10.1103/PhysRevB.92.195124 (2015).

21. Ma, D., Chen, H., Liu, H. \& Xie, X. C. Kondo effect with Weyl semimetal Fermi arcs. Phys. Rev. B 97, 045148, https://doi.org/10.1103/ PhysRevB.97.045148 (2018).

22. Chang, H.-R., Zhou, J., Wang, S.-X., Shan, W.-Y. \& Xiao, D. RKKY interaction of magnetic impurities in Dirac and Weyl semimetals. Phys. Rev. B 92, 241103, https://doi.org/10.1103/PhysRevB.92.241103 (2015).

23. Principi, A., Vignale, G. \& Rossi, E. Kondo effect and non-Fermi-liquid behavior in Dirac and Weyl semimetals. Phys. Rev. B 92, 041107, https://doi.org/10.1103/PhysRevB.92.041107 (2015).

24. Zheng, S.-H., Wang, R.-Q., Zhong, M. \& Duan, H.-J. Resonance states and beating pattern induced by quantum impurity scattering in Weyl/Dirac semimetals. Scientific Reports 6, 36106 EP- (2016).

25. Marques, Y. et al. Antibonding ground state of adatom molecules in bulk Dirac semimetals. Phys. Rev. B 96, 041112, https://doi. org/10.1103/PhysRevB.96.041112 (2017).

26. Friedel, J. Metallic alloys. Il Nuovo Cimento (1955-1965) 7, 287-311, https://doi.org/10.1007/BF02751483 (1958).

27. Marques, Y., Yudin, D., Shelykh, I. A. \& Seridonio, A. C. Resonant electron tunneling spectroscopy of antibonding states in a Dirac semimetal. Phys. Rev. B 97, 235121, https://doi.org/10.1103/PhysRevB.97.235121 (2018).

28. Plihal, M. \& Gadzuk, J. W. Nonequilibrium theory of scanning tunneling spectroscopy via adsorbate resonances: Nonmagnetic and Kondo impurities. Phys. Rev. B 63, 085404, https://doi.org/10.1103/PhysRevB.63.085404 (2001).

29. Haug, H. \& Jauho, A. Quantum Kinetics in Transport and Optics of Semiconductors. (Springer Series in Solid-State Sciences, Springer Berlin Heidelberg, 2008).

30. Bruus, H. \& Flensberg, K. Many-Body Quantum Theory in Condensed Matter Physics: An Introduction. (Oxford Graduate Texts, Oxford University Press, 2004).

31. Hubbard, J. Electron correlations in narrow energy bands. Proc. R. Soc. A 276, 238-257, https://doi.org/10.1098/rspa.1963.0204 (1963).

\section{Acknowledgements}

We thank the funding Brazilian agencies CNPq (Grant No. 307573/2015-0), CAPES, and São Paulo Research Foundation (FAPESP) Grant No. 2018/09413-0. I.A.S. acknowledges support from Horizon2020 project CoExAN, megagrant 14.Y26.31.0015 and Goszadanie no. 3.8884.2017/8.9 of the Ministry of Education and Science of Russian Federation. 


\section{Author Contributions}

Y.M., M.deS., M.S.F., I.A.S. and A.C.S. formulated the problem and wrote the manuscript. Y.M., W.N.M. and R.S.O. derived the expressions, performed their numerical computing and plotted the figures. All co-authors taken part in the discussions and reviewed the manuscript as well.

\section{Additional Information}

Supplementary information accompanies this paper at https://doi.org/10.1038/s41598-019-44842-8.

Competing Interests: The authors declare no competing interests.

Publisher's note: Springer Nature remains neutral with regard to jurisdictional claims in published maps and institutional affiliations.

(c) (i) Open Access This article is licensed under a Creative Commons Attribution 4.0 International License, which permits use, sharing, adaptation, distribution and reproduction in any medium or format, as long as you give appropriate credit to the original author(s) and the source, provide a link to the Creative Commons license, and indicate if changes were made. The images or other third party material in this article are included in the article's Creative Commons license, unless indicated otherwise in a credit line to the material. If material is not included in the article's Creative Commons license and your intended use is not permitted by statutory regulation or exceeds the permitted use, you will need to obtain permission directly from the copyright holder. To view a copy of this license, visit http://creativecommons.org/licenses/by/4.0/.

(c) The Author(s) 2019 